\title{
Fruit and vegetable consumption and risk of COPD: a prospective cohort study of men
}

\author{
Joanna Kaluza, ${ }^{1,2}$ Susanna C Larsson, ${ }^{1}$ Nicola Orsini, ${ }^{1}$ Anders Linden, ${ }^{3}$ Alicja Wolk ${ }^{1}$
}

\begin{abstract}
- Additional material is published online only. To view please visit the journal online (http://dx.doi.org/10.1136/ thoraxjn-2015-207851).

'Unit of Nutritional Epidemiology, Institute of Environmental Medicine Karolinska Institutet, Stockholm, Sweden

2Department of Human Nutrition, Warsaw University of Life Sciences-SGGW, Warsaw, Poland

${ }^{3}$ Unit for Lung and Airway Research, Institute of Environmental Medicine Karolinska Institutet, Stockholm, Sweden
\end{abstract}

Correspondence to Dr Joanna Kaluza, Department of Human Nutrition, Warsaw University of Life SciencesSGGW, 159C Nowoursynowska Str., Warsaw 02-776, Poland: joanna_kaluza@sggw.pl

Received 17 September 2015 Revised 7 November 2016 Accepted 8 November 2016 Published Online First 27 January 2017

\section{ABSTRACT}

Background Antioxidants present in fruits and vegetables may protect the lung from oxidative damage and prevent COPD.

Aims To determine the association between fruit and vegetable consumption and risk of COPD by smoking status in men.

Methods The population-based prospective Cohort of Swedish Men included 44335 men, aged 45-79 years, with no history of COPD at baseline. Fruit and vegetable consumption was assessed with a self-administered questionnaire.

Results During a mean follow-up of 13.2 years, 1918 incident cases of COPD were ascertained. A strong inverse association between total fruit and vegetable consumption and COPD was observed in smokers but not in never-smokers ( $p$-interaction $=0.02$ ). The agestandardised incidence rate per 100000 person-years in the lowest quintile ( $<2$ servings/day) of total fruit and vegetable consumption was 1166 in current smokers and 506 in ex-smokers; among those in the highest quintile ( $\geq 5.3$ servings/day), 546 and 255 per 100000 person-years, respectively. The multivariable HR of COPD comparing extreme quintiles of total fruit and vegetable consumption was 0.60 (95\% Cl 0.47 to 0.76 , p-trend $<0.0001)$ in current smokers and $0.66(95 \% \mathrm{Cl} 0.51$ to 0.85 , $p$-trend $=0.001$ ) in ex-smokers. Each one serving per day increment in total fruit and vegetable consumption decreased risk of COPD significantly by $8 \%$ $(95 \% \mathrm{Cl} 4 \%$ to $11 \%$ ) in current smokers and by $4 \%$ $(95 \% \mathrm{Cl} 0 \%$ to $7 \%$ ) in ex-smokers.

Conclusions These results indicate that high consumption of fruits and vegetables is associated with reduced COPD incidence in both current and ex-smokers but not in never-smokers.

\section{INTRODUCTION}

COPD is predicted by WHO to become the third cause of death worldwide. ${ }^{1}$ Oxidative stress induced by cigarette smoking is now recognised as a major predisposing factor in the pathogeneses of COPD. ${ }^{2}$ It was observed that antioxidant capacity in patients with COPD is substantially reduced as a result of cigarette smoking, with oxidative stress persisting long after the cessation of cigarette smoking, due to the continued endogenous production of reactive oxygen species. ${ }^{3}$ Thus, it can be hypothesised that high consumption of fruits and vegetables, a rich source of antioxidants, may protect the lung against oxidative damage and prevent COPD.
Key messages

What is the key question?

- Can fruit and vegetable consumption reduce risk of COPD?

What is the bottom line?

- High fruit and vegetable consumption is associated with lower risk of COPD in current and ex-smokers, but not in never-smokers.

\section{Why read on?}

- This study, using data from large population-based prospective cohort of men, allowed separate subgroup analyses of smokers and never-smokers due to high number of incident COPD cases. Current and ex-smokers who were consuming $\geq 5$ vs $<2$ servings per day of fruits and vegetables had $40 \%$ and $34 \%$ lower COPD risk, respectively.

Recent evidence indicates that diet may play an important role in COPD development. More specifically, a 'prudent' dietary pattern (ie, high consumption of fruits, vegetables, whole grain cereals and fish) in comparison with a 'Western' dietary pattern (ie, high consumption of white bread, processed meat, full-fat dairy products, sugar and chips) has been associated with a lower risk of impaired lung function and COPD. ${ }^{4-6}$ In a recently published prospective study, consumption of a healthy diet (high consumption of fruits, vegetables, whole grains, polyunsaturated fatty acids, nuts and long chain omega-3 fats and low consumption of red and processed meat, refined grains and sugar sweetened drinks consumption) was associated with a lower risk of COPD in US men and women.?

The potential association between fruit and vegetable consumption specifically and COPD mortality has previously been studied in only one prospective study of a mere 2917 men and 73 COPD-related deaths. ${ }^{8}$ In that study, an inverse association between consumption of fruits, but not vegetables, and COPD mortality was observed. Moreover, other observational studies have suggested that fruit consumption was inversely associated with chronic non-specific lung diseases ${ }^{9}$ and reduced cough with phlegm. ${ }^{10}$

Given the lack of large prospective studies on fruit and vegetable consumption and incidence of COPD, we examined this association in the Cohort 
of Swedish Men (COSM), a population-based prospective study with over 13 years of follow-up. We assessed whether smoking status, the main predictor of COPD, modified the association.

\section{METHODS}

\section{Study population}

The COSM was established in central Sweden in the late autumn of 1997. All men $(n=100303)$ born between 1918 and 1952 (45-79 years of age) and residing in Västmanland and Örebro counties received written information about the study and a self-administered questionnaire. Among the 48850 men who returned a completed questionnaire, we excluded those with missing or incorrect national identification numbers $(n=297)$, those who died prior to baseline $(n=55)$ and those with a previous cancer diagnosis (other than non-melanoma skin cancer) $(\mathrm{n}=2592)$. Moreover, men with a history of COPD (International Classification of Diseases and Related Health Problems, 10th Revision (ICD-10) code: J44) $(\mathrm{n}=281)$, those with implausible values for total energy intake ( $>3$ SDs from the mean value for log-transformed energy, $\mathrm{n}=567$ ) and those with missing data on fruit and vegetable consumption $(n=723)$ were excluded. The remaining analytical cohort of 44335 men was followed from 1 January 1998 through 31 December 2012. Both the original and analytical cohort well represented the Swedish male population in 1997 in terms of age distribution, educational level, prevalence of overweight and obesity, and smoking status compared with the Official Statistic of Sweden ${ }^{11-13}$ (see online supplementary table S1).

\section{Assessment of fruit and vegetable consumption and whole diet}

Data about food consumption were collected using a 96-item food frequency questionnaire (FFQ) in 1997. Men were asked to indicate how often, on average, they had consumed each type of food during the last year. Participants could choose from eight predefined frequency categories, ranging from never to three or more times per day. We defined the reported times eaten per day as the number of servings. The FFQ included 5 questions about fruit items (apples and pears; fresh and frozen berries; bananas; orange and citrus fruits; and other fruits) and 13 questions about vegetables (lettuce; spinach; cabbage; cauliflower; broccoli and Brussels sprouts; carrot; beetroot; pepper; tomatoes; onion and leek; garlic; green peas; and mixed frozen vegetables). We also combined specific vegetables into the following subgroups: green leafy vegetables (spinach and lettuce), cruciferous vegetables (cabbage, cauliflower, broccoli and Brussels sprouts) and root vegetables (carrots and beetroots).

The FFQ has been validated using four 1-week weighed dietary records every 3 months as the reference method. The Spearman correlation coefficients between estimates obtained from the FFQ and from the average of four 1-week dietary records ranged from 0.4 to 0.7 for individual fruit and vegetable items (Wolk, unpublished data).

We assessed dietary patterns based on two measurements of diet quality, that is, modified recommended food score (RFS) and non-RFS, which were developed and described previously. ${ }^{14}$ Based on dietary guidance, the modified RFS included 19 food items (compared with the original score we have omitted fruit and vegetable food items) that are recommended, that is, seven cereal products, five types of fish and seafood, three different low-fat dairy products, soya bean products, orange/grapefruit juice, nuts/almonds and olive oil. When each recommended food item was consumed at least one to three times per month or more, one point was assigned. A maximum score was 19 points, and higher score meant the better quality of diet. The non-RFS included 16 food items, that is, 3 unprocessed red meat items, 5 processed meat products, 3 high-fat dairy products, white bread, sweets, combined potato chips/popcorn and fried potatoes/French fries, mayonnaise and ice cream. Consumption of any of these non-recommended food items three times per week or more often was assigned one point, and if less often zero points. A maximum score was 16 points and was corresponding to the lowest quality of diet. ${ }^{14}$

\section{Other covariates}

The questionnaire also included information about education level, weight, height, smoking status, physical activity and alcohol consumption (type of alcoholic beverages and frequency of consumption). Body mass index (BMI) was calculated by dividing the weight $(\mathrm{kg})$ by the square of height $(\mathrm{m})$. Participants reported their level of activity at work, time spent on home/ housework, walking/bicycling and exercise as well as sedentary time per day including watching TV/reading and hours of sitting/lying down and sleeping. The time per day spent on each activity was multiplied by the activity's typical energy expenditure and expressed in metabolic equivalents. All individual activities were summed to calculate a metabolic equivalent-hours per day (24 hours) score (MET $\times$ hour/day). ${ }^{15}$ Men were asked about smoking status, including average number of cigarettes smoked per day at different ages (15-20, 21-30, 31-40, 41-50 and 51-60 years of age and current age), age they started smoking and, if applicable, age they stopped smoking. Pack-years were estimated from the smoking history by multiplying the number of years of smoking by the reported number of cigarettes smoked per day within respective age groups.

\section{Case ascertainment}

With the use of the unique personal identification number assigned to each Swedish resident, we identified incident COPD cases that were diagnosed in the cohort through linkage with the Swedish Patient Register (inpatient and outpatient registers) and the Swedish Cause of Death Register. Events of COPD were defined according to the ICD-10 (code J44). In the present study, a COPD case was defined as the first diagnosis of COPD (listed either as the primary diagnosis or at any diagnosis position) in the Swedish Patient Register or in the Cause of Death Register (only the primary position of COPD). A register study conducted in Sweden during 1999-2009 (a time period overlapping with our study) reported that incidence rates were increasing during those years. ${ }^{16}$ Furthermore, proportion of the cases diagnosed in primary care increased from $59 \%$ to $81 \%$ and patients were, an average, 7 years younger (66 vs 73 years old). These observations may indicate some degree of underdiagnosis during early years of follow-up of the COSM cohort.

\section{Statistical analysis}

Study participants were followed from 1 January 1998 to the date of the first COPD diagnosis, death or the end of the study follow-up period (31 December 2012), whichever occurred first. Total fruit and vegetable consumption, fruit consumption and vegetable consumption were each categorised into quintiles. Baseline characteristics of participants were standardised to the age distribution of the study population at baseline. Cox proportional hazards models were used to calculate HRs with $95 \%$ CIs of COPD. The age-adjusted and smoking-adjusted model included baseline age of participants (years, continuous) and their smoking status combined with pack-years of smoking (never; past $<20,20-39$ or $\geq 40$ pack-years; or current $<20$, 
20-39 or $\geq 40$ pack-years). The multivariable HRs were adjusted for the following potential confounders: education (less than high school, high school or university), BMI (<18.5, 18.5-24.9, $25-29.9$ or $\geq 30 \mathrm{~kg} / \mathrm{m}^{2}$ ), total physical activity (quintiles, MET $\times$ hour/day), energy intake (kcal/day, quintiles), alcohol intake (g/day, quintiles), modified RFS (scores, continuous) and non-RFS (scores, continuous). Furthermore, we examined a combined effect of total fruit and vegetable consumption with smoking status by including current smokers, ex-smokers, as well as never-smokers in the same model, where the reference group constituted never-smoking men in the highest quintile of total fruit and vegetable consumption. Missing data on educational level (0.7\%), smoking status (1.3\%), BMI (4.9\%) and total physical activity score $(22.7 \%)$ were modelled as separate categories. Fruit and vegetable consumption was mutually adjusted through inclusion in the same multivariable model. Also in the multivariable-adjusted analysis of specific type of fruits and vegetables, the individual fruit items and vegetable subgroups were included in the same model and mutually adjusted for each other.

The proportional hazards assumption was assessed by regressing scaled Schoenfeld residuals against survival time. There was no evidence of departure from the assumption. To calculate $p$ values for linear trend, the median values of each quintile category were used as a continuous variable. Using the likelihood ratio test, we tested statistical interaction on the multiplicative scale between fruit and vegetable consumption and smoking status in predicting the risk of COPD. Moreover, we examined the shape of the associations between total fruit and vegetables consumption, fruit consumption and vegetable consumption and risk of COPD using a restricted cubic-spline regression analysis with three knots (at the 10th, 50th and 90th percentile). ${ }^{17}$ Finally, we characterised the association between fruit and vegetable intake and occurrence of COPD in terms of disease-free survival time. Percentile differences, in years, were estimated for the fifth survival percentile (ie, difference in years by which $5 \%$ of the cohort experienced the event) using Laplace regression.

The statistical analyses were performed using SAS V.9.4 (SAS Institute, Cary, North Carolina, USA) and STATA V.13 (StataCorp, College Station, Texas, USA). All reported $\mathrm{p}$ values are two-sided; $\mathrm{p}$ values $\leq 0.05$ were considered statistically significant.

\section{RESULTS}

During a mean follow-up of 13.2 years (583 966 person-years, 1998-2012), we identified 1918 incident cases of COPD. The mean age of the participants was $60.2 \pm 9.7$ years, BMI was 25.8 $\pm 3.3 \mathrm{~kg} / \mathrm{m}^{2}$ and $62.8 \%$ of the men were ever-smokers $(24.3 \%$ current smokers, $38.5 \%$ ex-smokers). Men with higher total fruit and vegetable consumption were more likely to have completed a university education and were less likely to be current smokers (table 1). Compared with men in the lowest quintile of total fruit and vegetable consumption, those in the highest quintile had higher energy intake, alcohol consumption, as well as higher modified RFS and non-RFS. The Spearman rank correlation coefficient between fruit and vegetable consumption was 0.41 , and the median total fruit and vegetable consumption (expressed in servings per day) differed 4.7-fold between the highest and the lowest quintiles.

We observed strong inverse associations between fruit and vegetable consumption and risk of COPD (table 2). Men in the highest quintile of total fruit and vegetable consumption $(\geq 5.3$ servings/day) compared with the lowest quintile $(<2$ servings/ day) had a $35 \%$ (95\% CI 24\% to 45\%) lower risk of COPD. When we investigated fruits and vegetables separately, the risk of COPD incidence was $27 \%$ (95\% CI 15\% to 38\%) lower for fruit and $18 \%$ (95\% CI $3 \%$ to $30 \%$ ) lower for vegetable consumption among men in the highest compared with those in the lowest quintile. We performed a sensitivity analysis using the complete data set (32 363 participants; 1286 cases) and the results did not change substantially; the HRs of COPD for men in the highest versus the lowest quintile were 0.60 (95\% CI 0.49 to 0.74 ) for total fruits and vegetables, 0.72 (95\% CI 0.59 to 0.88 ) for fruits and $0.79(95 \%$ CI 0.64 to 0.97$)$ for vegetables.

To examine an influence of a potential underascertainment during early years of follow-up, we have calculated HRs stratified by time of diagnosis in 5-year periods (see online supplementary table S2). The observed results suggest that during the first years of follow-up COPD could be

Table 1 Age-standardised characteristics of the Cohort of Swedish Men $(n=44335)$

\begin{tabular}{|c|c|c|c|c|c|}
\hline & \multicolumn{5}{|c|}{ Quintiles (Q) of total fruit and vegetable consumption (servings/day) } \\
\hline & Q1 & Q2 & Q3 & Q4 & Q5 \\
\hline Total fruit and vegetable consumption, servings/day (median) & $<2.0(1.4)$ & $2.0-2.9(2.5)$ & $3.0-3.9(3.4)$ & $4.0-5.2(4.5)$ & $\geq 5.3(6.6)$ \\
\hline $\mathrm{n}$ & 8828 & 8846 & 8931 & 8837 & 8893 \\
\hline Age, mean (SD) & $61.2(9.9)$ & $60.1(9.6)$ & $59.5(9.7)$ & $59.7(9.6)$ & $60.2(9.6)$ \\
\hline University education, \% & 9.2 & 13.3 & 17.0 & 19.1 & 23.2 \\
\hline BMI, mean (SD) & $26.1(3.6)$ & $25.9(3.3)$ & $25.7(3.2)$ & $25.7(3.2)$ & $25.6(3.3)$ \\
\hline Total physical activity (METxhour/day), mean (SD) & $41.5(5.2)$ & $41.5(4.9)$ & $41.5(4.9)$ & $41.5(4.8)$ & $41.7(4.8)$ \\
\hline \multicolumn{6}{|l|}{ Smoking status } \\
\hline Current smokers, \% & 34.8 & 25.7 & 22.2 & 20.6 & 18.5 \\
\hline Ex-smokers, \% & 34.7 & 39.0 & 39.7 & 38.9 & 40.2 \\
\hline Never-smokers, \% & 28.6 & 33.9 & 36.8 & 39.4 & 40.4 \\
\hline Alcohol consumption (g/day), mean (SD) & $9.0(10.9)$ & $10.1(9.9)$ & $10.6(10.1)$ & $11.1(10.0)$ & $11.3(10.7)$ \\
\hline Energy intake (kcal/day), mean (SD) & $2361(790)$ & $2547(756)$ & $2653(782)$ & $2755(760)$ & $3032(901)$ \\
\hline Modified RFS (scores), mean (SD) & $8.0(2.9)$ & $9.8(2.8)$ & $10.5(2.9)$ & $11.1(2.9)$ & $11.4(3.0)$ \\
\hline Non-RFS (scores), mean (SD) & $3.7(1.4)$ & $3.9(1.4)$ & $4.0(1.5)$ & $4.1(1.5)$ & $4.2(1.8)$ \\
\hline
\end{tabular}


Table 2 HRs (95\% Cls) of COPD by quintiles of fruit and vegetables consumption in 44335 Swedish men, 1998-2012

\begin{tabular}{|c|c|c|c|c|c|c|}
\hline & \multicolumn{5}{|c|}{ Quintiles (Q), servings/day } & \multirow[b]{2}{*}{$p$ for trend } \\
\hline & Q1 & Q2 & Q3 & Q4 & Q5 & \\
\hline Total fruits and vegetables (median) & $<2.0(1.4)$ & $2.0-2.9(2.5)$ & $3.0-3.9(3.4)$ & $4.0-5.2(4.5)$ & $\geq 5.3(6.6)$ & \\
\hline No. of cases & 614 & 418 & 348 & 296 & 242 & \\
\hline Person-years & 110745 & 116687 & 119154 & 118657 & 118722 & \\
\hline Age-adjusted SIR per 100000 & 565 & 390 & 335 & 290 & 224 & \\
\hline Age-adjusted HR & 1.00 & $0.68(0.60-0.78)$ & $0.58(0.51-0.66)$ & $0.49(0.43-0.56)$ & $0.39(0.33-0.45)$ & $<0.0001$ \\
\hline Age-adjusted and smoking-adjusted HR & 1.00 & $0.79(0.69-0.89)$ & $0.75(0.66-0.85)$ & $0.66(0.57-0.75)$ & $0.55(0.47-0.64)$ & $<0.0001$ \\
\hline Multivariable $\mathrm{HR}^{*}$ & 1.00 & $0.86(0.76-0.98)$ & $0.84(0.74-0.97)$ & $0.76(0.65-0.88)$ & $0.65(0.55-0.76)$ & $<0.0001$ \\
\hline Fruit consumption (median) & $<0.5(0.3)$ & $0.5-0.7(0.6)$ & $0.8-1.1(1.0)$ & $1.2-1.8(1.5)$ & $\geq 1.9(2.5)$ & \\
\hline No. of cases & 536 & 417 & 344 & 338 & 283 & \\
\hline Person-years & 103166 & 124777 & 117483 & 119185 & 119355 & \\
\hline Age-adjusted SIR per 100000 & 580 & 402 & 328 & 303 & 237 & \\
\hline Age-adjusted HR & 1.00 & $0.67(0.59-0.76)$ & $0.55(0.48-0.62)$ & $0.50(0.44-0.58)$ & $0.39(0.34-0.46)$ & $<0.0001$ \\
\hline Age-adjusted and smoking-adjusted HR & 1.00 & $0.81(0.71-0.92)$ & $0.74(0.65-0.85)$ & $0.75(0.66-0.87)$ & $0.61(0.53-0.71)$ & $<0.0001$ \\
\hline Multivariable $\mathrm{HR}^{*} \dagger$ & 1.00 & $0.90(0.79-1.03)$ & $0.85(0.73-0.97)$ & $0.88(0.76-1.02)$ & $0.73(0.62-0.85)$ & 0.0002 \\
\hline Vegetable consumption (median) & $<1.2(0.8)$ & $1.2-1.8(1.6)$ & $1.9-2.5(2.2)$ & $2.6-3.5(3.0)$ & $\geq 3.6(4.6)$ & \\
\hline No. of cases & 578 & 418 & 368 & 299 & 255 & \\
\hline Person-years & 110402 & 116846 & 118041 & 119978 & 118698 & \\
\hline Age-adjusted SIR per 100000 & 508 & 387 & 354 & 290 & 251 & \\
\hline Age-adjusted HR & 1.00 & $0.76(0.67-0.86)$ & $0.69(0.60-0.78)$ & $0.56(0.49-0.65)$ & $0.48(0.42-0.56)$ & $<0.0001$ \\
\hline Age-adjusted and smoking-adjusted HR & 1.00 & $0.83(0.73-0.94)$ & $0.82(0.72-0.93)$ & $0.70(0.61-0.80)$ & $0.62(0.53-0.71)$ & $<0.0001$ \\
\hline Multivariable $\mathrm{HR}^{*} \dagger$ & 1.00 & $0.95(0.83-1.08)$ & $0.98(0.85-1.13)$ & $0.89(0.77-1.04)$ & $0.82(0.70-0.97)$ & 0.02 \\
\hline
\end{tabular}

underdiagnosed. To address a possible impact of underascertainment of COPD on observed risk estimates, we have excluded the first 5 years of follow-up; the HR of COPD incidence in the highest versus the lowest quintile was $0.61(95 \%$ CI 0.50 to 0.74 ) for total fruit and vegetables, 0.71 (95\% CI 0.59 to 0.86 ) for fruit and 0.78 (95\% CI 0.64 to 0.95$)$ for vegetable consumption.

\section{Findings by smoking status}

We investigated whether the association between fruit and vegetable consumption and risk of COPD varied by cigarette smoking status. Indeed, there was a statistically significant interaction for smoking status (never-smokers vs ever-smokers, $\mathrm{p}$ for interaction $=0.02$ ). Therefore, we presented results stratified by smoking status.

Among current smokers ( $\mathrm{n}=10780$ participants, 995 cases of COPD) and ex-smokers ( $n=17072$ participants, 747 cases of COPD), the age-standardised incidence rate (SIR) of COPD was lower among those who consumed $\geq 5.3$ servings/day of total fruits and vegetables (546 and 255 per 100000 person-years, respectively) than among those who consumed $<2.0$ servings/ day (1166 and 506 per 100000 person-years, respectively). Current and ex-smokers in the highest quintile of total fruit and vegetable consumption had a $40 \%$ (95\% CI 24\% to 53\%) and $34 \%$ (95\% CI $15 \%$ to $49 \%$ ), respectively, decreased risk of COPD compared with those in the lowest quintile (table 3 ). The HRs for extreme quintiles of fruit consumption in relation to COPD incidence were similar in current $(0.74,95 \%$ CI 0.58 to 0.93 ) and ex-smokers $(0.73,95 \%$ CI 0.57 to 0.94$)$, whereas vegetable consumption was significantly inversely associated with COPD risk only in current smokers $(0.69,95 \%$ CI 0.54 to
0.87), but not in ex-smokers $(0.92,95 \%$ CI 0.71 to 1.20$)$. Moreover, we investigated whether intake of fruits at various levels of vegetable intake and vice versa would have a different effect on COPD incidence (see online supplementary table S3). We found no evidence of interaction between fruit and vegetable consumption in current smokers ( $p$ value for interaction $=0.94$ ) or in ex-smokers ( $p$ value for interaction $=0.13$ ).

We investigated the shape of the association between total fruit and vegetable consumption and COPD risk by performing a restricted cubic spline analysis among current and ex-smokers. The risk of COPD was decreasing with increasing consumption of total fruits and vegetables per day (figure 1). Each one serving per day increment in total fruit and vegetable consumption was associated with $8 \%(95 \%$ CI $4 \%$ to $11 \%)$ decreased risk of COPD in current smokers and with 4\% (95\% CI 0\% to $7 \%$ ) in ex-smokers. For fruit consumption (figure 2), the risk of COPD was non-significantly decreasing for each one serving increment in consumption (HR $0.95,95 \%$ CI 0.88 to 1.03 in current smokers and $0.95,95 \%$ CI 0.87 to 1.03 in ex-smokers). Vegetable consumption was inversely associated with the risk of COPD only in current smokers; each one serving increase in vegetable consumption was associated with a 9\% (HR 0.91, 95\% CI 0.86 to 0.95 ) reduction in COPD risk (figure 3). There was no indication of non-linearity in the observed associations (all $\mathrm{p}$ non-linearity $>0.11$, figures $1-3$ ).

We also investigated whether specific fruits and vegetables were associated with risk of COPD in smokers (table 4). In this analysis, we combined current and ex-smokers in one group to increase statistical power of the analysis. Consumption of apples or pears, green leafy vegetables and peppers was inversely associated with COPD; for each daily serving increment in the 
Table 3 HRs ( $95 \%$ Cls) of COPD by quintiles of fruit and vegetable consumption by smoking status in Swedish men, 1998-2012

\begin{tabular}{|c|c|c|c|c|c|c|}
\hline & \multicolumn{5}{|c|}{ Quintiles (Q) } & \multirow[b]{2}{*}{$p$ for trend } \\
\hline & Q1 & Q2 & Q3 & Q4 & Q5 & \\
\hline \multicolumn{7}{|c|}{ Total fruit and vegetable consumption, servings/day (median) } \\
\hline & $<2.0(1.4)$ & $2.0-2.9(2.5)$ & $3.0-3.9(3.4)$ & $4.0-5.2(4.5)$ & $\geq 5.3(6.6)$ & \\
\hline \multicolumn{7}{|l|}{ Ever-smokers } \\
\hline No. of cases/no. of men & $577 / 6115$ & $375 / 5720$ & $316 / 5537$ & $267 / 5257$ & $207 / 5223$ & \\
\hline Age-adjusted SIR per 100000 & 803 & 559 & 508 & 450 & 336 & \\
\hline Multivariable HRt & 1.00 & $0.83(0.73-0.95)$ & $0.83(0.72-0.96)$ & $0.75(0.64-0.88)$ & $0.62(0.52-0.73)$ & $<0.0001$ \\
\hline \multicolumn{7}{|l|}{ Current smokers } \\
\hline No. of cases/no. of men & $376 / 3031$ & 219/2273 & $163 / 2003$ & 139/1829 & 98/1644 & \\
\hline Age-adjusted SIR per 100000 & 1166 & 884 & 765 & 715 & 546 & \\
\hline Multivariable HRt & 1.00 & $0.83(0.70-0.99)$ & $0.80(0.66-0.97)$ & $0.73(0.59-0.90)$ & $0.60(0.47-0.76)$ & $<0.0001$ \\
\hline \multicolumn{7}{|l|}{ Ex-smokers } \\
\hline No. of cases/no. of men & $201 / 3084$ & $156 / 3447$ & $153 / 3534$ & $128 / 3428$ & $109 / 3579$ & \\
\hline Age-adjusted SIR per 100000 & 506 & 374 & 380 & 326 & 255 & \\
\hline Multivariable HR ${ }^{\dagger}$ & 1.00 & $0.84(0.68-1.04)$ & $0.89(0.71-1.10)$ & $0.78(0.62-0.99)$ & $0.66(0.51-0.85)$ & 0.001 \\
\hline \multicolumn{7}{|l|}{ Never-smokers } \\
\hline No. of cases/no. of men & $60 / 5537$ & & $30 / 3281$ & $25 / 3479$ & $32 / 3596$ & \\
\hline Age-adjusted SIR per 100000 & 84 & & 77 & 63 & 70 & \\
\hline Multivariable HR & 1.00 & & $1.05(0.67-1.65)$ & $0.82(0.51-1.34)$ & $1.03(0.64-1.64)$ & 0.78 \\
\hline \multicolumn{7}{|c|}{ Fruit consumption, servings/day (median) } \\
\hline & $<0.5(0.3)$ & $0.5-0.7(0.6)$ & $0.8-1.1(1.0)$ & $1.2-1.8(1.5)$ & $\geq 1.9(2.5)$ & \\
\hline \multicolumn{7}{|l|}{ Ever-smokers } \\
\hline No. of cases/no. of men & $508 / 5866$ & $374 / 6166$ & $318 / 5496$ & $298 / 5211$ & $244 / 5113$ & \\
\hline Age-adjusted SIR per 100000 & 789 & 564 & 506 & 472 & 372 & \\
\hline Multivariable $\mathrm{HR}^{*} \dagger$ & 1.00 & $0.87(0.76-1.00)$ & $0.87(0.75-1.01)$ & $0.88(0.76-1.03)$ & $0.73(0.62-0.86)$ & 0.001 \\
\hline \multicolumn{7}{|l|}{ Current smokers } \\
\hline No. of cases/no. of men & $336 / 2909$ & $212 / 2517$ & $182 / 2030$ & $149 / 1689$ & $116 / 1635$ & \\
\hline Age-adjusted SIR per 100000 & 1133 & 854 & 820 & 805 & 582 & \\
\hline Multivariable $\mathrm{HR}^{*} \dagger$ & 1.00 & $0.88(0.73-1.04)$ & $0.93(0.77-1.12)$ & $0.93(0.76-1.14)$ & $0.74(0.58-0.93)$ & 0.03 \\
\hline \multicolumn{7}{|l|}{ Ex-smokers } \\
\hline No. of cases/no. of men & $172 / 2957$ & $162 / 3649$ & $136 / 3466$ & $149 / 3522$ & $128 / 3478$ & \\
\hline Age-adjusted SIR per 100000 & 503 & 398 & 341 & 335 & 282 & \\
\hline Multivariable $\mathrm{HR}^{*} \dagger$ & 1.00 & $0.87(0.70-1.08)$ & $0.81(0.64-1.02)$ & $0.84(0.67-1.07)$ & $0.73(0.57-0.94)$ & 0.02 \\
\hline \multicolumn{7}{|l|}{ Never-smokers } \\
\hline No. of cases/no. of men & $54 / 5140$ & & $23 / 3238$ & $36 / 3647$ & $34 / 3868$ & \\
\hline Age-adjusted SIR per 100000 & 86 & & 60 & 78 & 66 & \\
\hline Multivariable $H R^{*}$ & 1.00 & & $0.69(0.42-1.14)$ & $0.90(0.58-1.40)$ & $0.73(0.45-1.16)$ & 0.35 \\
\hline \multicolumn{7}{|c|}{ Vegetable consumption, servings/day (median) } \\
\hline & $<1.2(0.8)$ & $1.2-1.8(1.6)$ & $1.9-2.5(2.2)$ & $2.6-3.5(3.0)$ & $\geq 3.6(4.6)$ & \\
\hline \multicolumn{7}{|l|}{ Ever-smokers } \\
\hline No. of cases/no. of men & $539 / 5873$ & $385 / 5686$ & $326 / 5475$ & $268 / 5409$ & $224 / 5409$ & \\
\hline Age-adjusted SIR per 100000 & 748 & 577 & 525 & 439 & 373 & \\
\hline Multivariable $\mathrm{HR}^{*} \dagger$ & 1.00 & $0.93(0.81-1.07)$ & $0.93(0.80-1.08)$ & $0.86(0.73-1.01)$ & $0.78(0.65-0.93)$ & 0.004 \\
\hline \multicolumn{7}{|l|}{ Current smokers } \\
\hline No. of cases/no. of men & $354 / 2790$ & $211 / 2250$ & $174 / 2016$ & $146 / 1928$ & $110 / 1796$ & \\
\hline Age-adjusted SIR per 100000 & 1136 & 864 & 810 & 713 & 585 & \\
\hline Multivariable $\mathrm{HR}^{*} \dagger$ & 1.00 & $0.83(0.69-0.99)$ & $0.83(0.68-1.01)$ & $0.81(0.65-1.00)$ & $0.69(0.54-0.87)$ & 0.003 \\
\hline \multicolumn{7}{|l|}{ Ex-smokers } \\
\hline No. of cases/no. of men & $185 / 3083$ & $174 / 3436$ & $152 / 3459$ & $122 / 3481$ & $114 / 3613$ & \\
\hline Age-adjusted SIR per 100000 & 447 & 415 & 379 & 307 & 286 & \\
\hline Multivariable $\mathrm{HR}^{*} \dagger$ & 1.00 & $1.11(0.89-1.38)$ & $1.11(0.88-1.39)$ & $0.94(0.73-1.21)$ & $0.92(0.71-1.20)$ & 0.26 \\
\hline \multicolumn{7}{|l|}{ Never-smokers } \\
\hline No. of cases/no. of men & $54 / 5856$ & & $37 / 3282$ & 29/3406 & $27 / 3349$ & \\
\hline Age-adjusted SIR per 100000 & 69 & & 94 & 75 & 67 & \\
\hline Multivariable $\mathrm{HR}^{*}$ & 1.00 & & $1.64(1.06-2.54)$ & $1.39(0.86-2.26)$ & $1.33(0.79-2.24)$ & 0.29 \\
\hline
\end{tabular}


Current smokers

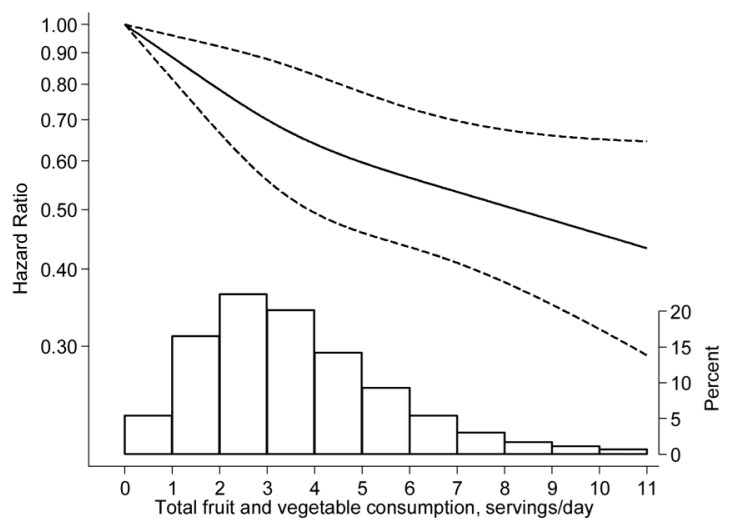

Ex-smokers

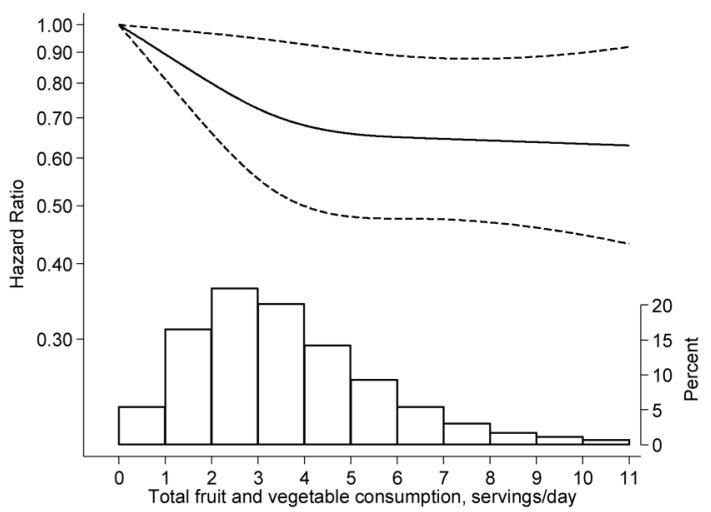

Figure 1 Multivariable-adjusted HR (solid line) of COPD incidence as a function of total fruit and vegetable consumption among current smokers ( $p$ for non-linearity=0.28) and ex-smokers ( $p$ for non-linearity=0.11). The long dashed lines represent $95 \%$ Cls. The distribution of total fruit and vegetable consumption is presented at the bottom of the figure as a histogram.
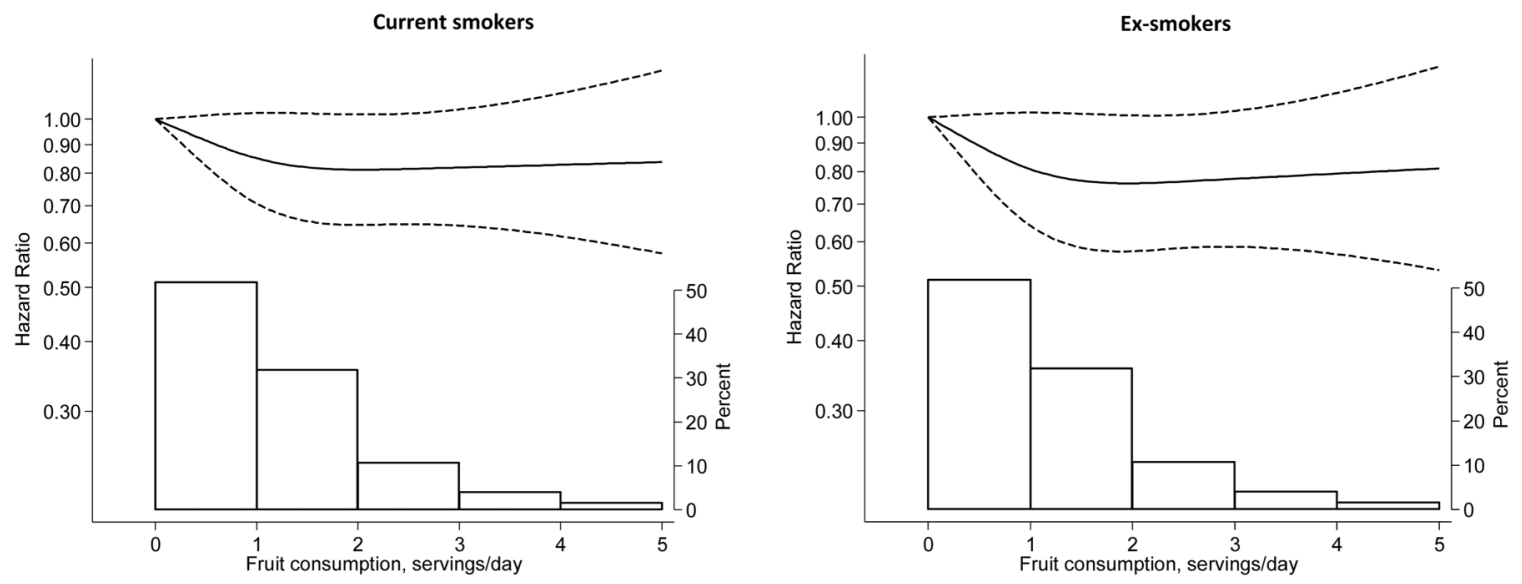

Figure 2 Multivariable-adjusted HR (solid line) of COPD incidence as a function of fruit consumption among current smokers ( $p$ for non-linearity $=0.16$ ) and ex-smokers ( $p$ for non-linearity $=0.15$ ). The long dashed lines represent $95 \% \mathrm{Cls}$. The distribution of fruit consumption is presented at the bottom of the figure as a histogram.
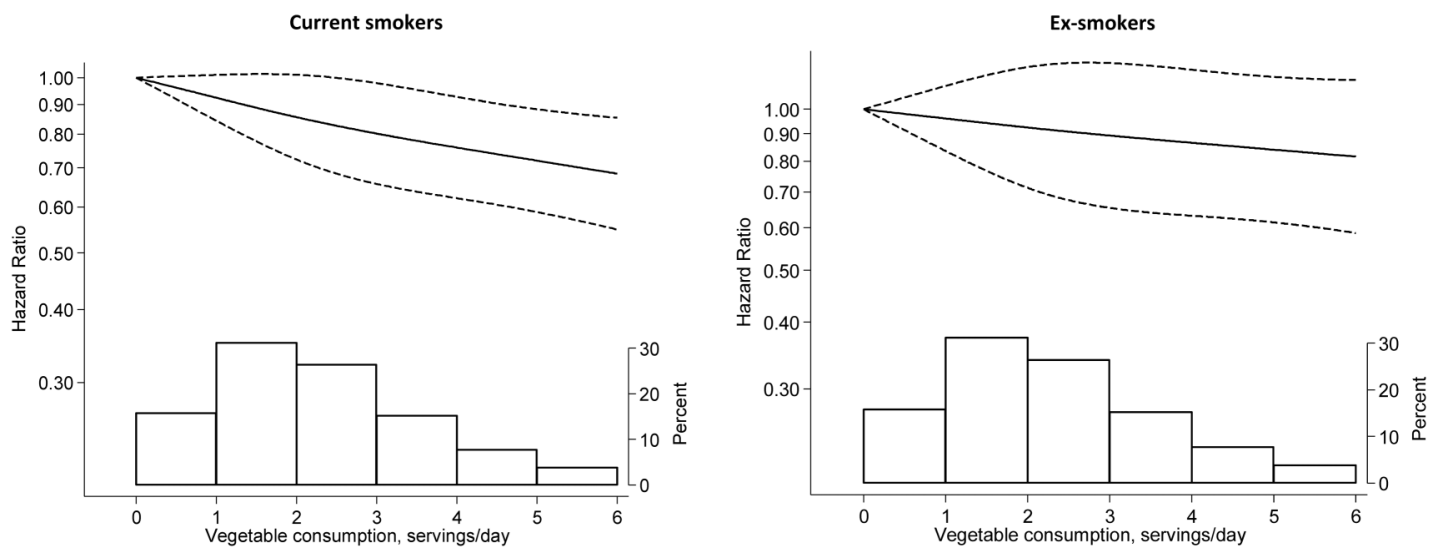

Figure 3 Multivariable-adjusted HR (solid line) of COPD incidence as a function of vegetable consumption among current smokers ( $p$ for non-linearity=0.67) and ex-smokers ( $p$ for non-linearity=0.92). The long dashed lines represent $95 \% \mathrm{Cls}$. The distribution of vegetable consumption is presented at the bottom of the figure as a histogram.

intake of these foods, the risk of COPD decreased by $23 \%$ (95\% CI $9 \%$ to $34 \%$ ), $32 \%$ (95\% CI $13 \%$ to $47 \%$ ) and $39 \%$ (95\% CI $13 \%$ to $57 \%$ ), respectively. Consumption of berries, bananas, citrus fruits, cruciferous vegetables, root vegetables, tomatoes, onions, garlic and green peas was not significantly associated with COPD.

To examine the association of combined effect of total fruit and vegetable consumption with smoking status, we conducted 
Table 4 HRs ( $95 \%$ Cls) of COPD by categories of subgroups of fruit and vegetables consumption in 27852 ever-smokers, 1998-2012

\begin{tabular}{|c|c|c|c|c|}
\hline & \multicolumn{3}{|l|}{ Categories } & \multirow[b]{2}{*}{$p$ for trend } \\
\hline & \multicolumn{3}{|c|}{ Fruit consumption } & \\
\hline Apples or pears, servings/day (median) & $0(0)$ & $0.1-0.9(0.2)$ & $\geq 1.0(1.0)$ & \\
\hline No. of cases/no. of men & $130 / 1424$ & $1250 / 20866$ & $203 / 4174$ & \\
\hline Multivariable $\mathrm{HR}^{*}$ & 1.00 & $0.88(0.72-1.08)$ & $0.70(0.54-0.90)$ & 0.003 \\
\hline Berries, servings/day (median) & $0(0)$ & $0.1-0.9(0.1)$ & $\geq 1.0(1.0)$ & \\
\hline No. of cases/no. of men & $193 / 2845$ & $1207 / 21049$ & $34 / 1653$ & \\
\hline Multivariable $\mathrm{HR}^{*}$ & 1.00 & $1.13(0.95-1.34)$ & $0.85(0.58-1.25)$ & 0.08 \\
\hline Bananas, servings/day (median) & $0(0)$ & $0.1-0.9(0.5)$ & $\geq 1.0(1.0)$ & \\
\hline No. of cases/no. of men & $135 / 1735$ & $1343 / 22357$ & $134 / 2435$ & \\
\hline Multivariable $\mathrm{HR}^{*}$ & 1.00 & $1.01(0.84-1.23)$ & $1.14(0.88-1.48)$ & 0.63 \\
\hline Citrus fruits, servings/day (median) & $0(0)$ & $0.1-0.9(0.1)$ & $\geq 1.0(1.0)$ & \\
\hline No. of cases/no. of men & $235 / 2881$ & $1113 / 20460$ & $100 / 1477$ & \\
\hline Multivariable $\mathrm{HR}^{*}$ & 1.00 & $0.92(0.78-1.08)$ & $1.02(0.79-1.33)$ & 0.22 \\
\hline \multicolumn{5}{|l|}{ Vegetable consumption } \\
\hline Green leafy vegetables, servings/day (median) $\dagger$ & $<0.2(0.1)$ & $0.2-0.9(0.4)$ & $\geq 1.0(1.1)$ & \\
\hline No. of cases/no. of men & $884 / 10264$ & $755 / 14746$ & $103 / 2842$ & \\
\hline Multivariable $\mathrm{HR}^{*}$ & 1.00 & $0.86(0.77-0.97)$ & $0.62(0.49-0.79)$ & 0.0001 \\
\hline Cruciferous vegetables, servings/day (median) $\ddagger$ & $<0.2(0.1)$ & $0.2-0.9(0.3)$ & $\geq 1.0(1.2)$ & \\
\hline No. of cases/no. of men & $829 / 10454$ & $836 / 15832$ & $77 / 1566$ & \\
\hline Multivariable $\mathrm{HR}^{*}$ & 1.00 & $1.05(0.94-1.18)$ & $1.32(1.01-1.72)$ & 0.19 \\
\hline Root vegetables, servings/day (median)§ & $<0.2(0.1)$ & $0.2-0.9(0.4)$ & $\geq 1.0(1.1)$ & \\
\hline No. of cases/no. of men & $643 / 9658$ & $936 / 15698$ & $163 / 2496$ & \\
\hline Multivariable $\mathrm{HR}^{*}$ & 1.00 & $0.96(0.86-1.07)$ & $0.96(0.79-1.16)$ & 0.89 \\
\hline Peppers, servings/day (median) & $0(0)$ & $0.1-0.9(0.2)$ & $\geq 1.0(1.0)$ & \\
\hline No. of cases/no. of men & $360 / 3975$ & $1039 / 19675$ & $29 / 964$ & \\
\hline Multivariable $\mathrm{HR}^{*}$ & 1.00 & $1.00(0.87-1.15)$ & $0.63(0.42-0.96)$ & 0.02 \\
\hline Tomatoes, servings/day (median) & $0(0)$ & $0.1-0.9(0.2)$ & $\geq 1.0(1.0)$ & \\
\hline No. of cases/no. of men & $174 / 2098$ & $1191 / 20493$ & $162 / 3349$ & \\
\hline Multivariable $\mathrm{HR}^{*}$ & 1.00 & $0.86(0.72-1.01)$ & $0.96(0.76-1.22)$ & 0.77 \\
\hline Onions, servings/day (median) & $0(0)$ & $0.1-0.9(0.5)$ & $\geq 1.0(1.0)$ & \\
\hline No. of cases/no. of men & $79 / 1002$ & $1426 / 23797$ & $62 / 1256$ & \\
\hline Multivariable $\mathrm{HR}^{*}$ & 1.00 & $1.07(0.84-1.36)$ & $0.98(0.68-1.43)$ & 0.93 \\
\hline Garlic, servings/day (median) & $0(0)$ & $0.1-0.9(0.1)$ & $\geq 1.0(1.0)$ & \\
\hline No. of cases/no. of men & $560 / 6959$ & $804 / 16574$ & $36 / 670$ & \\
\hline Multivariable $\mathrm{HR}^{*}$ & 1.00 & $0.93(0.82-1.05)$ & $0.91(0.64-1.31)$ & 0.79 \\
\hline Green peas, servings/day (median) & $0(0)$ & $0.1-0.9(0.1)$ & $\geq 1.0(1.0)$ & \\
\hline No. of cases/no. of men & $212 / 2903$ & $1285 / 22357$ & $19 / 355$ & \\
\hline Multivariable $\mathrm{HR}^{*}$ & 1.00 & $0.96(0.82-1.13)$ & $1.11(0.66-1.88)$ & 0.27 \\
\hline \multicolumn{5}{|c|}{$\begin{array}{l}\text { *Adjusted for age (years, continuous), education (less than high s } \\
\text { (MET×hour/day, quintiles), pack-years of smoking }(<20,20-39 \text { or } \\
\text { food score (scores, continuous) and non-recommended food score } \\
\text { tIncluding lettuce and spinach. } \\
\text { łIncluding cabbage, cauliflower, broccoli and Brussels sprouts. } \\
\text { §lncluding carrot and beetroot. } \\
\text { MET, metabolic equivalent of energy expenditure (kcal/kg×hour). }\end{array}$} \\
\hline
\end{tabular}

an analysis including current, ex-smokers, as well as neversmokers in the same model (figure 4). Compared with neversmokers with high ( $\geq 5.3$ servings/day) total fruit and vegetable consumption (the reference group), current and ex-smokers with low consumption ( $<2$ servings/day) had a 13.5 -fold and sixfold higher risk of COPD, and those with high consumption had a 7.5 -fold and a 3.6-fold higher risk, respectively.

To address time aspect in terms of COPD-free survival time related to total fruit and vegetable consumption, we have performed Laplace regression (see online supplementary figure S1). Compared with current and ex-smokers with consumption of 0 servings/day of fruits and vegetables the first 5\% of COPD events among those who consumed 5 servings/day occurred around 1.6 and 1.25 years later, respectively, while among those who consumed 8 servings/day about 2.0 and 1.75 years later, respectively.

We did not observe statistically significant associations between fruit and vegetable consumption and COPD risk among never-smokers.

\section{DISCUSSION}

In this population-based prospective cohort of men, we observed that consumption of fruits and vegetables was inversely associated with COPD incidence. High total fruit and vegetable consumption ( $\geq 5.3$ servings/day) as compared with low consumption ( $<2$ servings/day) was associated with reduced risk of COPD by $40 \%$ in current smokers and by $34 \%$ in ex-smokers. No association was observed in never-smokers. The 


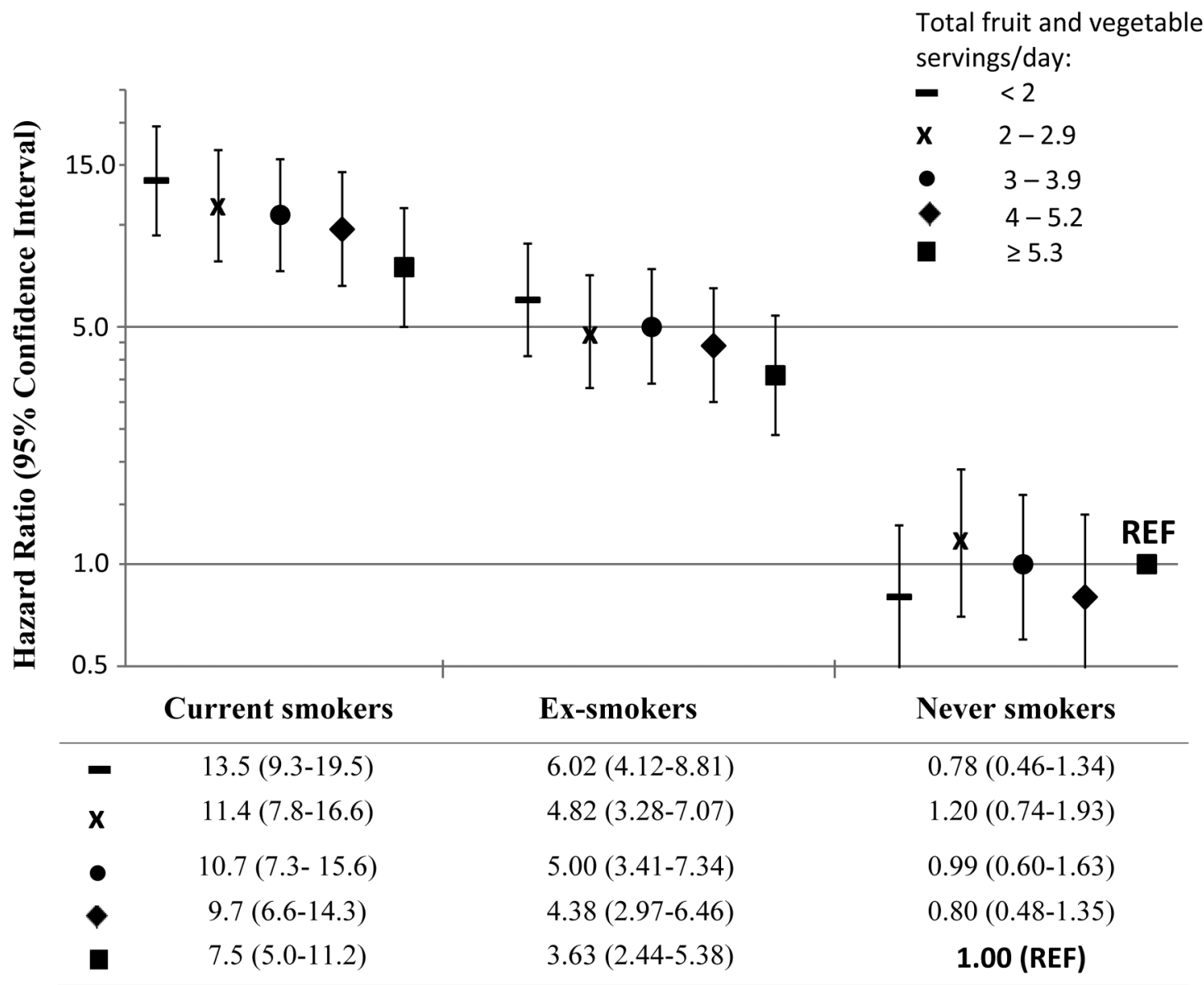

Figure $4 \mathrm{HRs}(95 \% \mathrm{Cls})$ of COPD by quintiles of total fruit and vegetable consumption and smoking status ( $p$ for interaction=0.02). HRs were adjusted for age, education, body mass index, physical activity, intake of energy, alcohol consumption and modified recommended food score and non-recommended food score. REF, reference.

risk of COPD in smokers with total fruit and vegetable consumption $<2$ servings/day was 13.5 -fold increased in current and sixfold in ex-smokers compared with never-smokers with high consumption; in those with $\geq 5.3$ servings/day, it was increased by 7.5 -fold (current smokers) and by 3.6 -fold (ex-smokers).

Findings from this first prospective study on total fruit and vegetable consumption and COPD incidence are in line with a 3 -year intervention in patients with COPD showing improved lung function after the increased consumption ${ }^{18}$ and also with cross-sectional studies of lung functions (better values of $\mathrm{FEV}_{1}$ and $\mathrm{FVC}^{19}$ and $\mathrm{FEV}^{20}$ among people with higher fruit and vegetable consumption).

Our findings for fruits only are in agreement with the previous small cohort study on COPD mortality (the highest vs lowest tertile HR $0.49,95 \mathrm{CI} 0.35$ to 0.96$)^{8}$ and with majority of cross-sectional studies on COPD. ${ }^{21}$ The inverse association with vegetables in the present study was observed previously only in minority of cross-sectional studies on COPD, ${ }^{21}$ but not in the prospective study of COPD mortality. ${ }^{8}$ The observed inverse association with apple/pears consumption is in agreement with a case-control study of COPD. ${ }^{22}$ Furthermore, Butland $e t a l^{23}$ reported that higher apple consumption was associated with better lung function in a cross-sectional analysis, but not in a longitudinal analysis. The observed inverse associations in our study with peppers and with green leafy vegetables, but not with other specific vegetables and fruits, may be due to rare intake or lack of variation in consumption of the latter.
Our results are also in line with findings of prior reports on healthy diet and COPD, which used the Alternate Healthy Eating Index-2010 (AHEI-2010, where 1/3 of the score is based on fruit and vegetable consumption $)^{7}$ and dietary patterns (where $1 / 2$ of a prudent pattern is based on fruit and vegetable consumption) ${ }^{45}$ to assess the quality of diet. People with the highest AHEI-2010 compared with those with the lowest had a $33 \%$ lower risk of COPD, ${ }^{7}$ and men and women in the highest quintile of the prudent pattern compared with those in the lowest quintile had a $50 \%$ and $33 \%$, respectively, lower COPD incidence. $^{45}$

It is known that the pathogenesis of COPD may involve oxidative stress and inflammation. ${ }^{24}$ Potent proinflammatory compounds trigger local tissue remodelling that can impair lung function over long periods of time. ${ }^{24}$ There is evidence that these processes are intense among smokers. ${ }^{25-27}$ There are also data indicating that the impaired antioxidant status correlates with increased severity of COPD. ${ }^{28} 29$ In particular, higher plasma lipid peroxidation has been associated with higher risk for progression in patients with COPD, while higher catalase and erythrocyte glutathione activities were associated with decreased risk of progression of this disease. ${ }^{29}$ Some studies, but not all, ${ }^{30}$ have found significant associations of high fruit and vegetable consumption with reduced levels of oxidative stress ${ }^{31}$ and inflammation parameters ${ }^{32}$ as well as with increased levels of antioxidant defence. ${ }^{31}$ It has been shown that intake of fruits and vegetables rich in vitamin $C, \beta$-carotene, flavonoids and other antioxidants is inversely correlated not only with oxidative 
stress parameters (urinary 8-iso prostaglandin $F 2 \alpha$ and F2-isoprostane) but also with inflammatory biomarkers (such as $C$ reactive protein, interleukin- 6 , tumour necrosis factor- $\alpha$ and 15 -keto-dihydro-PGF2 $\alpha$ metabolite). ${ }^{32}$ It has been observed that vitamin $\mathrm{C}, \beta$-carotene and vitamin $\mathrm{E}$ intake correlate positively with lung function. ${ }^{33-35}$ The reason why the inverse association of fruit and vegetable consumption with COPD was restricted to current and ex-smokers could plausibly be due to higher oxidative stress in these groups and continued endogenous production of reactive oxygen species even after smoking cessation. Among never-smokers, the pathogenic mechanisms leading to COPD may be different from those in smokers and ex-smokers; these may relate more to genetic predisposition and environmental exposures triggering lower oxidative stress and inflammation than does tobacco smoke.

Strengths of the current study include the population-based and prospective design. Large number of incident COPD cases allowed separate analyses in current smokers, ex-smokers and never-smokers. Detailed information on diet and the available data on potential risk factors for COPD also allowed extensive adjustment for confounding.

Our study has some limitations as in all observational studies. Estimates of fruit and vegetable consumption and other factors were based on self-reports assessed at baseline only and the exposures could also be changed during the follow-up, potentially leading to misclassification. However, the FFQ used in this study had a relatively high validity. Moreover, consumption of fruits and vegetables in the studied cohort was relatively stable between 1997 and 2009 (increased only by 0.7 serving/ week). ${ }^{36}$ Nevertheless, any misclassification of fruit and vegetable consumption, because of the prospective design, would be non-differential and most likely have attenuated rather than exaggerated the true associations. Unmeasured or residual confounding cannot be completely ruled out, although we have adjusted for major potential confounders.

Given that COPD as an entity was launched during the $1970 \mathrm{~s}^{37}$ and that the awareness among healthcare professionals continued to evolve during the 1990 s and later, ${ }^{16}$ we cannot rule out underascertainment of COPD in the early years of follow-up. Underascertainment of cases may lead to attenuation of risk estimates. ${ }^{38}$ Indeed, we observed that risk estimates for the first years of follow-up were attenuated in comparison to the later period. However, by excluding the first 5 years of follow-up, we found that the associations remained similar. In general, it is possible that men who are more health conscious may also be more likely to seek medical care. Nevertheless, because of the healthcare system in Sweden, all inhabitants have the same affordable access to medical care; however, in certain socioeconomic or educational groups with less healthcareseeking behaviour, an overestimation of the association between fruit and vegetable consumption and COPD incidence may have occurred. Moreover, we cannot rule out that some patients classified as having COPD had obtained the diagnosis without the correct spirometry assessment, even though this investigation is formally required for a clinical diagnosis of COPD. Ideally, new studies in this research area should include this type of verifying spirometry that would presumably further strengthen the validity of the case definition.

In conclusion, in this prospective cohort study of men, high fruit and vegetable consumption was inversely associated with COPD incidence among smokers, but not among non-smokers. The present findings confirm the strong impact of cigarette smoking on the development of COPD and also indicate that diet rich in fruit and vegetables may have an important role in prevention of COPD. Nevertheless, non-smoking and smoking cessation remain the main public health message to prevent development of COPD.

Contributors JK had full access to all data in the study and takes responsibility for the integrity of the data analysis. JK, SCL, AL and AW contributed with study concept and design. JK and NO performed the statistical analysis. All authors helped to write the manuscript and performed critical revision of the manuscript for important intellectual content.

Funding This study was supported by a research grant from the Swedish Research Council/Infrastructure (AW), the Karolinska Institutet's Distinguished Professor Award $(\mathrm{AW})$ and the Swedish Heart-Lung Foundation (AL) and from King Gustav V's and Queen Victoria's Freemason Research Foundation (AL). Federal funding was obtained in accordance with the ALF and LUA agreement, from Stockholm County Council $(\mathrm{AL})$ and the Västra Götaland Region (AL).

Competing interests None declared.

Ethics approval The study was approved by the Regional Ethical Review Board at Karolinska Institutet (Stockholm, Sweden).

Provenance and peer review Not commissioned; externally peer reviewed.

\section{REFERENCES}

1 WHO. Cause-specific mortality. Estimates for 2000-2012. Health statistics and information systems. http://www.who.int/healthinfo/global_burden_disease/ estimates/en/index1.html

2 Kirkham PA, Barnes PJ. Oxidative stress in COPD. Chest 2013;144:266-73.

3 Louhelainen N, Rytilä P, Haahtela T, et al. Persistence of oxidant and protease burden in the airways after smoking cessation. BMC Pulm Med 2009;9:25.

4 Varraso R, Fung TT, Hu FB, et al. Prospective study of dietary patterns and chronic obstructive pulmonary disease among US men. Thorax 2007;62:786-91.

5 Varraso R, Fung TT, Barr RG, et al. Prospective study of dietary patterns and chronic obstructive pulmonary disease among US women. Am J Clin Nutr 2007;86:488-95.

6 Shaheen SO, Jameson KA, Syddall HE, et al. The relationship of dietary patterns with adult lung function and COPD. Eur Respir J 2010;36:277-84.

7 Varraso R, Chiuve SE, Fung TT, et al. Alternate Healthy Eating Index 2010 and risk of chronic obstructive pulmonary disease among US women and men: Prospective study. BMJ 2015;350:h286.

8 Walda IC, Tabak C, Smit HA, et al. Diet and 20-year chronic obstructive pulmonary disease mortality in middle-aged men from three European countries. Eur J Clin Nutr 2002;56:638-43

9 Miedema I, Feskens EJM, Heederik D, et al. Dietary determinants of long-term incidence of chronic nonspecific lung diseases. Am J Epidemiol 1993;138:37-45.

10 Butler LM, Koh WP, Lee HP, et al. Dietary fiber and reduced cough with phlegm: a cohort study in Singapore. Am J Respir Crit Care Med 2004;170:279-87.

11 Official Statistics of Sweden. Statistisk årsbok 1999, Statistical Yearbook of Sweden 1999. Vol 85. Örebro; Statistics Sweden, 1999.

12 Official Statistics of Sweden: Health and Medical Care. Statistisk årsbok 2010 Statistical Yearbook of Sweden, 2010.

13 Johansson SE, Qvist J. Fetma en tickande bomb också i Sverige? (Obesity-a ticking bomb even in Sweden?) Välfärdsbulletinen 1998;4:14-16.

14 Kaluza J, Håkansson N, Brzozowska A, et al. Diet quality and mortality: a population-based prospective study of men. Eur J Clin Nutr 2009;63:451-7.

15 Norman A, Bellocco R, Bergström A, et al. Validity and reproducibility of self-reported total physical activity--differences by relative weight. Int I Obes Relat Metab Disord 2001;25:682-8.

16 Stallberg B, Janson C, Johansson G, et al. Management, morbidity and mortality of COPD during an 11-year period: an observational retrospective epidemiological register study in Sweden (PATHOS). Prim Care Respir J 2014;23:38-45.

17 Harrell FE Jr., Lee KL, Pollock BG. Regression models in clinical studies: determining relationships between predictors and response. J Natl Cancer Inst 1988:80:1198-202.

18 Keranis E, Makris D, Rodopoulou P, et al. Impact of dietary shift to higher-antioxidant foods in COPD: a randomised trial. Eur Respir J 2010;36:774-80.

19 Okubo H, Shaheen SO, Ntani G, et al. Processed meat consumption and lung function: Modification by antioxidants and smoking. Eur Respir J 2014;43:972-82.

20 Tabak C, Smit HA, Rasanen L, et al. Dietary factors and pulmonary function: a cross sectional study in middle aged men from three European countries. Thorax 1999;54:1021-6.

21 Boeing $H$, Bechthold A, Bub A, et al. Critical review: vegetables and fruit in the prevention of chronic diseases. Eur J Nutr 2012;51:637-63.

22 Watson L, Margetts B, Howarth P, et al. The association between diet and chronic obstructive pulmonary disease in subjects selected from general practice. Eur Respir J 2002;20:313-18.

23 Butland BK, Fehily AM, Elwood PC. Diet, lung function, and lung function decline in a cohort of 2512 middle age men. Thorax 2000;55:102-8. 
24 Domej W, Oettl K, Renner W. Oxidative stress and free radicals in COPDimplications and relevance for treatment. Int J Chron Obstruct Pulmon Dis 2014;9:1207-24.

25 Bloomer RJ. Decreased blood antioxidant capacity and increased lipid peroxidation in young cigarette smokers compared to nonsmokers: impact of dietary intake. Nutr J 2007;6:39.

26 Chin YW, Chuang HY, Huang MC, et al. Comparison of plasma antioxidant levels and related metabolic parameters between smokers and non-smokers. Kaohsiung J Med Sci 2009;25:423-9.

27 Andersson A, Bossios A, Malmhäll C, et al. Effects of tobacco smoke on IL-16 in $\mathrm{CD} 8^{+}$cells from human airways and blood: a key role for oxygen free radicals? AJP-Lung Cell Mol Physiol 2011;300:L43-55.

28 Ahmad A, Shameem M, Husain Q. Altered oxidant-antioxidant levels in the disease prognosis of chronic obstructive pulmonary disease. Int J Tuberc Lung Dis 2013:17:1104-9.

29 Arja C, Surapaneni KM, Raya P, et al. Oxidative stress and antioxidant enzyme activity in South Indian male smokers with chronic obstructive pulmonary disease. Respirology 2013;18:1069-75.

30 Baldrick FR, Elborn JS, Woodside JV, et al. Effect of fruit and vegetable intake on oxidative stress and inflammation in COPD: a randomised controlled trial. Eur Respir J 2012;39:1377-84.
31 Rink SM, Mendola P, Mumford SL, et al. Self-report of fruit and vegetable intake that meets the 5 a day recommendation is associated with reduced levels of oxidative stress biomarkers and increased levels of antioxidant defense in premenopausal women. J Acad Nutr Diet 2013;113:776-85.

32 Holt EM, Steffen LM, Moran A, et al. Fruit and vegetable consumption and its relation to markers of inflammation and oxidative stress in adolescents. J Am Diet Assoc 2009;109:414-21.

33 Schwartz J, Weiss ST. Relationship between dietary vitamin C intake and pulmonary function in the First National Health and Nutrition Examination Survey (NHANES I). Am J Clin Nutr 1994;59:110-14.

34 Schwartz J, Weiss ST. Dietary factors and their relation to respiratory symptoms. The Second National Health and Nutrition Examination Survey. Am J Epidemiol. 1990;132:67-76.

$35 \mathrm{Hu} \mathrm{G}$, Cassano PA. Antioxidant nutrients and pulmonary function: The Third National Health and Nutrition Examination Survey (NHANES III). Am J Epidemiol 2000;151:975-81.

36 Olofsson C, Discacciati A, Åkesson A, et al. Changes in fruit, vegetable and juice consumption after the diagnosis of type 2 diabetes: a prospective study in men. Br J Nutr. Zoblished :Onthe.First: 13 Jul 2016. doi:10.1017/S0007114516002257

37 Cohen BH, Ball WC Jr, Brashears S, et al. Risk factors in chronic obstructive pulmonary disease (COPD). Am J Epidemiol 1977;105:223-32.

38 Rothman KJ. Epidemiology: an introduction. Oxford University Press, 2012. 